Proc. of the 15th Int. Workshop on Slow Positron Beam Techniques and Applications, Prague, September 2-6, 2019

\title{
Positron Beam Facility at NEPOMUC: Status and Recent Developments
}

\author{
C. Hugenschmidt \\ Technische Universität München, FRM II, Lichtenbergstr. 1, 85748 Garching, Germany
}

\begin{abstract}
Low-energy positron beams are applied in a number of experiments in solid state physics and materials science as well as in atomic and particle physics. These experiments comprise of surface studies, depth dependent defect spectroscopy, and fundamental research with leptonic systems. The neutron induced positron source in Munich (NEPOMUC) is operated as a user facility providing a positron beam with an intensity of about $10^{9}$ moderated positrons per second. In this paper, a review of the status of the positron beam facility with its instruments is given and several experiments are exemplarily highlighted. Recent and future developments for further improving the performance of positron beam experiments are discussed.
\end{abstract}

DOI: 10.12693/APhysPolA.137.145

PACS/topics: 78.70.Bj, 41.75.Fr, 36.10.Dr

\section{Introduction}

Intense positron sources, i.e., sources providing more than $10^{7}$ monoenergetic positrons per second, are operated at several large scale facilities worldwide. Based on the pair-production process such positron sources have been implemented either at electron linacs at AIST [1] and KEK [2] (both located in Tsukuba, Japan) and at ELBE, Germany [3] or at research reactors in Delft, Netherlands [4] and at NEPOMUC at FRM II Munich [5].

Since more than fifteen years the high-intensity positron source NEPOMUC is operated as a user facility at the Technical University Munich. The primary beam of NEPOMUC provides about $10^{9}$ positrons per second at an energy of $1 \mathrm{keV}$. Most of the measurements, however, are carried out by using the brightness enhanced low-energy positron beam $(20 \mathrm{eV})$ yielding an intensity of about $5 \times 10^{7}$ remoderated positrons per second. At present, five different instruments are connected to the positron beam line allowing experiments in various fields in physical research and materials science.

So far, numerous depth dependent positron beam studies have been performed in order to gain information of lattice defects near the surface or to image defect distributions using a scanning positron beam. The high positron beam intensity was shown to be beneficial for considerably reducing the measurement time e.g., for coincident Doppler-broadening spectroscopy (CDBS) and for improving the signal-to-noise ratio for various applications such as positron annihilation lifetime spectroscopy (PALS). Moreover, the high beam brightness allowed the realization of novel experimental techniques such as timedependent positron annihilation induced Auger-electron spectroscopy (PAES) for surface studies with topmost

e-mail: christoph.hugenschmidt@frm2.tum.de layer sensitivity, production of the positronium negative ion $\mathrm{Ps}^{-}$or first attempts towards a positron-electron pair plasma. Another advantage of the high initial beam intensity is the possibility to realize positron microbeams by using multiple remoderation for brightness enhancement.

In the following the generation and preparation of the (remoderated) positron beam at NEPOMUC is briefly reviewed before the status of the positron beam facility and its instrumentation is given. Details of the positron beam instruments as well as experimental highlights are presented followed by a discussion of recent developments.

\section{Generation of the positron beam}

The reactor based positron source NEPOMUC at FRM II provides the world's strongest positron beam with an intensity of $10^{9}$ moderated positrons per second [6]. Based on the neutron capture reaction ${ }^{113} \mathrm{Cd}(\mathrm{n}, \gamma){ }^{114} \mathrm{Cd}$ high-energy prompt $\gamma$ radiation is released which eventually generates positrons by production in a $\mathrm{Pt}$ foil structure. Due to its negative positron work function of about $2 \mathrm{eV}$, (self-)moderated positrons are emitted at the Pt surface. By applying positive high voltage the so-called primary positron beam is extracted with a kinetic energy of $1 \mathrm{keV}$ and adiabatically guided in a longitudinal magnetic field of $40-70 \mathrm{mT}$ via an optional remoderation unit to various experiments.

In fact, for most positron beam applications an enhanced beam brightness, i.e., higher phase space density, is beneficial allowing the reduction of the beam spot to about $250 \mu \mathrm{m}$ at the samples position for spatial resolved Doppler broadening measurements or the improvement of the time resolution at PALS. For this reason, a W(100) single crystal is applied as positron remoderator in back reflection geometry [7]. At the entrance of this device the $1 \mathrm{keV}$ positron beam is released from the magnetic guiding field by a field termination consisting of metallic glass stripes mounted inside a $\mu$-metal flange before being 
focused onto the $\mathrm{W}$ crystal by an electric lens and a magnetic short-focus lens. After separation from the primary beam with a magnetic dipole field perpendicular to the beam axis the low-energy (usually $20 \mathrm{eV}$ ) remoderated beam is injected into the magnetic guiding field of the beam line by using several electrodes. The remoderation setup was shown to yield an efficiency of about $5 \%$. In addition, two beam switches allow for toggling between the primary and the remoderated positron beam. Most recently, the system was upgraded in order to enable quick replacement of the moderation crystal without breaking the beamline vacuum and to allow for exact positioning of the crystal surface within the focus of the magnetic lens (see M. Dickmann, et al., these proceedings [8]).

For characterization of the beam parameters, i.e., intensity and shape, remote controlled beam monitors basically consisting of micro-channel plates with phosphor screen and mirror can be inserted into the beamline. The diameter of the remoderated beam was determined to be $<2 \mathrm{~mm}$ (FWHM) in a $6 \mathrm{mT}$ guiding field.

\section{Positron beam instruments}

The beam line with (basic pressure $<5 \times 10^{-8}$ mbar) leads from the remoderator unit via a beam switch to a platform in the experimental hall of the FRM II. As shown in Fig. 1, five instrument stations are connected to the positron beam facility for various applications.

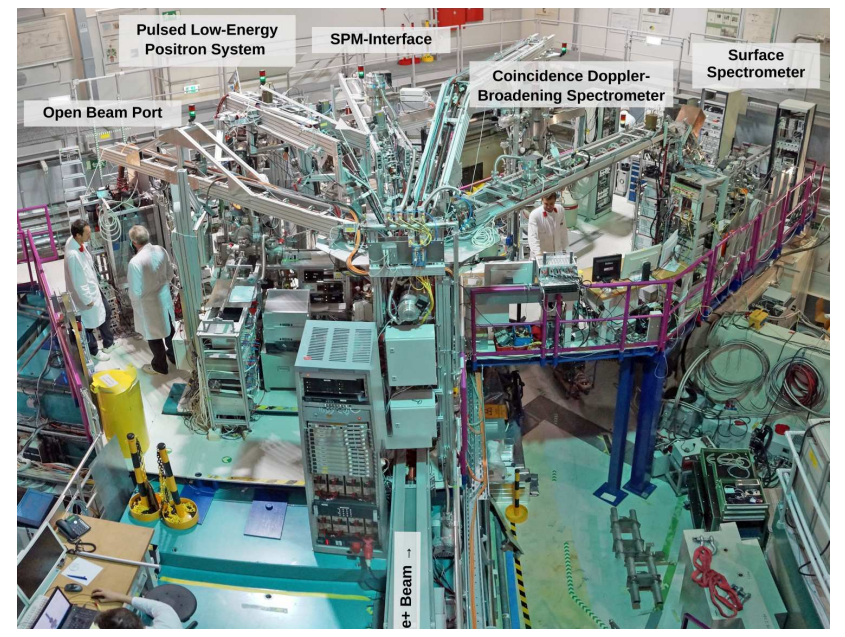

Fig. 1. View onto the positron beam facility at NEPOMUC. The (remoderated) positron beam passes a beam switch to various instruments: open beam port (OP) for short-term experiments such as the present magnetic dipole trap for pair plasma studies, pulsed lowenergy positron system (PLEPS), interface for a scanning positron microscope (SPM), coincidence Doppler broadening spectrometer (CDBS), the surface spectrometer for positron annihilation induced Auger electron spectroscopy (PAES). Currently a new positron diffractometer is put into operation (not shown) and a buffer gas trap is planned to be installed in the straight section upstream the beam switch.

\subsection{Spatial resolved defect spectroscopy}

In materials science the analysis of vacancy-like defects (PALS and DBS) and the chemical environment around such defects (CDBS) plays an important role. The application of depth dependent defect spectroscopy for the investigation of e.g., depth profiles or thin films, however, requires either continuous positron beams for (coincident) Doppler broadening spectroscopy or pulsed beams for positron lifetime measurements (see below). Depth dependent DBS is performed by variation of the positron implantation energy and detection of the annihilation quanta with a single high-purity Ge detector. Information about the chemical surrounding of open volume defects or precipitates near the surface, in thin layers or in the bulk can be gained by coincident read out of a pair of Ge detectors (CDBS).

The CDB spectrometer at NEPOMUC is equipped with four high purity Ge detectors (30\% efficiency). The positron implantation energy can be varied between 0.1 and $30 \mathrm{keV}$, and lateral scanning with the positron beam within an area of $19 \times 19 \mathrm{~mm}^{2}$ is implemented by using piezo positioners. Hence 3D information about the defect distribution of the sample can be gained for the investigation of e.g., ion irradiated alloys [9] or the local oxygen vacancy concentration in single crystalline $\mathrm{YBa}_{2} \mathrm{Cu}_{3} \mathrm{O}_{7-\delta}$ thin films [10].

For CDBS with increased spatial resolution a positron microbeam has been developed based on threefold positron moderation. For this purpose, the CDB spectrometer has been upgraded with an additional transmission type remoderator (100 nm thin $\mathrm{Ni}(100)$ foil) allowing measurements with a minimum beam diameter of $33 \mu \mathrm{m}$. Three positron beam monitors located upstream the spectrometer, at the position of the remoderation foil and at the sample position are used for optimization of the beam setting. Due to the high beam intensity provided by NEPOMUC high-resolution 2D images of defect distributions obtained by DBS can be recorded within a measurement time of about $160 \mathrm{~min} / \mathrm{mm}^{2}$ with a step width of $50 \mu \mathrm{m}$. Recently, the distribution of vacancylike defects and precipitates around laser beam welds has been investigated successfully [11].

The pulsed low energy positron system PLEPS enables depth dependent positron lifetime measurements using a pulsed beam of variable positron implantation energy between 0.5 and $20 \mathrm{keV}$. Depending on the used detector for providing the stop-signal the acquisition rate is in the order of $10^{4}$ counts per second with a total timeresolution of 180-240 ps. Typically the recording time for lifetime spectra containing several $10^{6}$ counts amounts to a few minutes only with a peak-to-background ratio of better than $10^{5}$. The time-window is routinely set to $40 \mathrm{~ns}$ but can also be extended to $160 \mathrm{~ns}$ for accurate measurements of long lifetimes.

Examples of PLEPS studies are the investigation of the free volume in polymer thin films [12], vacancies in layers of perovskite oxides [13], defects in palladium films after 
hydrogen loading [14], or in photovoltaic thin layers [15]. More recently, PALS under laser illumination has been carried out providing valuable insight into the change in the defect charge states in Mg-implanted GaN [16].

For future PALS with micrometer resolution the SPM, which is equipped with a further remoderation unit, has to be connected to the NEPOMUC beam line via an appropriate interface. Recently, this interface comprising pulsing units, an additional positron remoderator, and a so-called energy elevator have been successfully put into operation [17].

\subsection{Surface experiments with positron beams}

Positrons can be applied to study both the elemental composition and the structure of surfaces with sensitivity to the topmost atomic layer (see e.g., review [18]). Lowenergy positron beams of a few eV were shown to be well suited for the elemental analysis of surfaces using PAES. Compared to conventional AES induced by keV-electrons or X-rays, PAES exhibits several advantages such as exceptional surface sensitivity due to positron surface states and suppressed secondary electron background in the range of Auger-transition energies. At NEPOMUC PAES is performed at the surface spectrometer which is equipped with an electron gun and an X-ray source to enable the complementary examination of the sample surface by conventional AES or photoelectron spectroscopy. Thanks to the high beam intensity time dependent PAES has become possible for the first time. A prominent experimental example is the in situ observation of the theoretically predicted surface segregation process in CuPd [19]. Due to space limitations in the experimental areas, however, the PAES setup is currently disconnected from the beamline in order to enable the operation of a new positron diffractometer in its immediate vicinity.

As counterpart to high-energy electron diffraction (RHEED), (total) reflection high-energy positron diffraction ((T)RHEPD) is considered as a powerful tool to reveal the structure of surfaces, i.e., the structure of the topmost atomic layer and the layer immediately below it. TRHEPD can be applied to study e.g., phase transitions at surfaces, lattice vibrations in the topmost surface layer, as well as tiny distortions of the surface structure. Followed by the pioneering work of Kawasuso et al. [20] the only TRHEPD apparatus in operation worldwide is located at the slow positron facility at KEK in Tsukuba. Taking advantage of the high positron beam intensity we constructed a novel positron diffractometer with optional transmission-type $\mathrm{Ni}(100)$ remoderator in order to further reduce the measurement time and to increase the signal-to-noise ratio. Due to the high required beam energy of $10-30 \mathrm{keV}$, the positrons have to be accelerated accordingly and the sample has to be kept on high potential (inside a Faraday cage) during the measurement. First TRHEPD experiments are planned to be performed in the beginning of 2020 .

\subsection{Fundamental research with positrons and electrons}

At the open beam port (OP) additional experimental setups can be flanged to the positron beam line. In the past, low-energy positrons have been used to e.g., test of time-of-flight system for PAES, to moderate positrons in a nitrogen filled drift chamber, or to study reemitted cold positronium. A highlight has been the implementation of a tabletop tandem accelerator for the production and detection of the positronium negative ion $\mathrm{Ps}^{-}$. This device allowed us to measure the $\mathrm{Ps}^{-}$decay rate with unprecedented accuracy yielding a value of $2.0875(50) \mathrm{ns}^{-1}$ in agreement with higher-order quantum electrodynamics calculations [21].

At present, a magnetic dipole trap basically comprising of a permanent magnet with $E \times B$ filter for particle injection and various diagnostic tools is connected to the positron beam line. Within the APEX collaboration various experiments have been conducted in order to reach the goal to confine and study a neutral positron-electron plasma for the first time. The injection efficiency of a low-energy positron beam into the confinement volume of the dipole trap has been demonstrated to be $100 \%$ [22]. In order to avoid positron loss on supporting materials it is currently planned to install a so-called levitated dipole device for the creation of an electron-positron plasma.

\section{Summary and outlook}

At present, three different experiments at NEPOMUC are in routine operation and open for external users: PLEPS for depth dependent PALS, CDBS for the spectroscopy and imaging of defects, and the dipole trap for pair plasma experiments at the OP. At the SPM interface last components are currently optimized and the new positron diffractometer will be put into operation within the next reactor cycle. In order to overcome the space limitation of the instruments it is planned to extend the positron beam line into a new experimental hall, commissioning of which is still pending, immediately adjacent to the reactor building. This will open the field for various major experimental innovations: We plan to combine PAES and TRHEPD with a common sample preparation chamber in order to study both the elemental composition and the surface structure with topmost layer sensitivity of samples under identical conditions. In addition, depth dependent measurements of the angular correlation of annihilation radiation (ACAR) will become possible by connecting our 2D-ACAR spectrometer to the NEPOMUC beam line. This will allow us to study the evolution of the electronic structure from the surface to the bulk for the first time. Furthermore, within a collaboration of the University of California, San Diego, University of Greifswald, Max-Planck Institute for Plasma Physics, and Technical University Munich we plan to install a buffer gas trap at NEPOMUC in order to 
turn the steady-state beam into high-density pulses. By including a multi-cell trap for positron accumulation the ultimate goal would be to deliver unprecedented ultraintense short pulses containing up to $10^{12}$ positrons.

\section{Acknowledgments}

The author thanks all members of the NEPOMUC team, the collaborators from the University of the Bundeswehr Munich (lifetime experiments), and the APEX-collaboration (pair plasma project), as well as numerous external users for their great efforts in pursuing positron beam experiments. Financial support from the DFG (projects HU978/15-1 and HU978/16-1) and from the BMBF (projects 05K13WO1 and 05K16WO7) is gratefully acknowledged.

\section{References}

[1] R. Suzuki, T. Ohdaira, T. Mikado, A. Uedono, H. Ohgaki, T. Yamazaki, S. Tanigawa, Mater. Sci. Forum 114, 255 (1997).

[2] T. Hyodo, K. Wada, A. Yagishita, et al., J. Phys. Conf. Ser. 262, 012026 (2011).

[3] R. Krause-Rehberg, G. Brauer, M. Jungmann, A. Krille, A. Rogov, K. Noack, Appl. Surf. Sci. 255, 22 (2008)

[4] H. Schut, A. van Veen, J. de Roode, F. Labohm, Mater. Sci. Forum 444, 507 (2004).

[5] C. Hugenschmidt, B. Löwe, J. Mayer, C. Piochacz, P. Pikart, R. Repper, M. Stadlbauer, K. Schreckenbach, Nucl. Instrum. Methods Phys. Res. A 593, 616 (2008).

[6] C. Hugenschmidt, H. Ceeh, T. Gigl, F. Lippert, C. Piochacz, P. Pikart, M. Reiner, J. Weber, S. Zimnik, J. Phys. Conf. Ser. 443, 012079 (2013).

[7] C. Piochacz, G. Kögel, W. Egger, C. Hugenschmidt, J. Mayer, K. Schreckenbach, P. Sperr, M. Stadlbauer, G. Dollinger, Appl. Surf. Sci. 255, 98 (2008).
[8] M. Dickmann, W. Egger, G. Kögel, S. Vohburger, C. Hugenschmidt, Acta Phys. Pol. A 137, 149 (2020).

[9] R.M. Hengstler-Eger, P. Baldo, L. Beck, et al., J. Nucl. Mater. 423, 170 (2012).

[10] M. Reiner, T. Gigl, R. Jany, G. Hammerl, C. Hugenschmidt, Appl. Phys. Lett. 106, 111910 (2015).

[11] T. Gigl, L. Beddrich, M. Dickmann, B. Rienäcker, M. Thalmayr, S. Vohburger, C. Hugenschmidt, New J. Phys. 19, 123007 (2017).

[12] W. Egger, P. Sperr, G. Kögel, M. Wetzel, H.J. Gudladt, Appl. Surf. Sci. 255, 209 (2008).

[13] D.J. Keeble, R.A. Mackie, W. Egger, B. Löwe, P. Pikart, C. Hugenschmidt, T.J. Jackson, Phys. Rev. B 81, 064102 (2010).

[14] J. Cizek, I. Prochazka, O. Melikhova, et al., Phys. Status Solidi C 6, 2364 (2009).

[15] A. Uedono, M.M. Islam, T. Sakurai, C. Hugenschmidt, W. Egger, R. Scheer, R. Krause-Rehberg, K. Akimoto, Thin Solid Films 603, 418 (2016).

[16] A. Uedono, S. Takashima, M. Edo, et al., Phys. Status Solidi B 255, 1700521 (2018).

[17] M. Dickmann, J. Mitteneder, G. Kögel, W. Egger, P. Sperr, U. Ackermann, C. Piochacz, G. Dollinger, Nucl. Instrum. Methods Phys. Res. A 821, 40 (2016).

[18] C. Hugenschmidt, Surf. Sci. Rep. 71, 547 (2016).

[19] J. Mayer, C. Hugenschmidt, K. Schreckenbach, Phys. Rev. Lett. 105, 207401 (2010).

[20] A. Kawasuso, S. Okada, Phys. Rev. Lett. 81, 2695 (1998).

[21] H. Ceeh, C. Hugenschmidt, K. Schreckenbach, S.A. Gärtner, P.G. Thirolf, F. Fleischer, D. Schwalm, Phys. Rev. A 84, 062508 (2011).

[22] E.V. Stenson, S. Nißl, U. Hergenhahn, Phys. Rev. Lett. 121, 235005 (2018). 